\title{
Was Sie über den Umgang mit diesem Nachschlagewerk wissen sollten
}

- Es gibt unterschiedliche Sortierungssystematiken. Wir gehen folgendermaßen vor: Zusammengesetzte Substantive bzw. Bindestrich-Begriffe (Air-Sea, Check-in, Check-out time, E-Mail-Kennzahlen) und Abkürzungen mit Zeichen (Ü/F) werden eingeordnet, als ob sie zusammengeschrieben werden. Begriffe aus mehreren Wörtern und ohne Bindestrich (à la carte, à discretion, en deux) werden eingeordnet, als ob es getrennte Begriffe sind.

- Pfeile innerhalb der Texte sind Querverweise und machen aufmerksam auf inhaltlich zusammenhängende Begriffe.

- Die bei den Begriffen aufgeführte Literatur verweist nicht nur auf die jeweiligen Quellenangaben. Es sind mitunter Werke hinzugefügt, die die entsprechende Thematik vertiefen.

- Im Anhang haben wir insbesondere für Studierende Literatur zusammengestellt, die einen Einstieg in die Welt des Tourismus, der Hotellerie sowie der Gastronomie erleichtern und erste Orientierung geben.

- Jeder Begriff ist am Ende mit den Initialen der Autorin, des Autors bzw. der Autoren gekennzeichnet (abw; bk; nsc/mp). Der im Anhang befindliche Autorenindex nennt die Autorin, den Autor bzw. das Autorenteam. Am Ende des Buches sind die Kurzbiographien aufgeführt, die mit dem Autorenindex verbunden sind.

- Aus Gründen der besseren Lesbarkeit wurde auf das,Gendern' (m/w/d) verzichtet. Inhalte und Hinweise in den Ausführungen richten sich selbstverständlich an alle Geschlechter gleichermaßen. 
